\title{
308
}

\section{MODELLING OF CAVITATION IN A RECIPROCATING PLUNGER PUMP}

\author{
Kevin EDGE, Clifford BURROWS and Neil LECKY-THOMPSON
}

Fluid Power Centre

School of Mechanical Engineering

University of Bath

Bath, BA2 7AY, UK

\begin{abstract}
This paper reports on the use of a commercial CFD package to model the flow fields and bubble trajectories in the cylinder of a reciprocating plunger pump during the suction stroke. The predicted behaviour of bubbles is compared with results obtained from a previously-reported flow visualisation study of a mock pump. The good qualitative agreement provides the basis for using the CFD model to examine the effect of changes to the pump valve and cylinder geometries.
\end{abstract}

\section{KEYWORDS}

Plunger pump, Cavitation, CFD

\section{INTRODUCTION}

Reciprocating plunger pumps with self-acting valves are commonly used in applications where the fluid is corrosive, as in many process plants, or where it has high contamination levels, as is common in mining installations. In these pumps the control of flow into and out of individual cylinders is usually achieved by means of pressure-actuated spring-loaded non-return valves as shown schematically in Figure 1. A study of the effect of these self-acting valve characteristics on the dynamic performance of these pumps has been reported by Johnston et al [1.]. They demonstrated the significance of correctly selecting the stiffness of both the suction and delivery springs to minimise the likelihood of cavitation and to maximise the pumping efficiency.

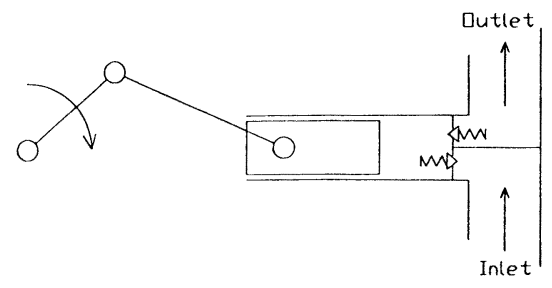

Figure 1. Valve configuration 
It is possible, during the suction stroke, for the fluid velocity in the vicinity of the inlet valve seat to be sufficiently high for the pressure to fall to the vapour pressure of the fluid. Cavitation then occurs and the cavitation bubbles are swept into the cylinder. The collapse of the cavities occurs near the end of the suction stroke or at the beginning of the delivery stroke. Severe damage can occur if bubbles collapse in the vicinity of the boundary walls, therefore it is important to be able to assess the effect of valve and cylinder geometries to minimise the likelihood of cavitation erosion.

The study of cavitation during the suction stroke in piston pumps does not appear to have received much attention by researchers. Related studies on poppet valves have been conducted by, for example, Oshima [2.], Ishii et al [3.] and Ueno et al [4.] although these studies were undertaken under steady flow conditions.

In an earlier paper [5.] the authors presented a preliminary flow visualisation study of water cavitation in a mock reciprocating pump cylinder snown in Figure 2.



Figure 2. Mock cylinder

A spring-loaded poppet valve was used to emulate the pump suction valve and the rig was configured to represent the behaviour of a single cylinder pump. It was possible using high-speed photography to identify locations in the cylinder of cavity growth and collapse at different times in the suction stroke, but no evidence was found of cavities collapsing near boundaries. It was thought that this may have been due to the symmetrical configuration of the mock cylinder and there was a need to design a rig to visualise cavitation in a real pump cylinder.
The limited strength of the perspex chamber meant that the mock pump could not be used to investigate behaviour when cavities are present in the cylinder at the start of the delivery stroke. Also the available lighting source and the restricted nature of the test circuit only allowed experiments to be carried out at low pumping speed and for a single valve configuration.

These various limitations led to the decision to undertake a CFD study of the problem.

\section{SOFTWARE}

At the time of the study the available software package [6.] was not capable of modelling vaporous cavitation, therefore it could not be used to undertake a quantitative study of bubble formation and behaviour in the mock pump. The package did, however, allow the solution of Lagrangian differential equations governing mass, momentum and energy conservation for a two-phase flow consisting of a dispersed phase within a continuous one.

It was thought that this could be used to provide qualitative information about bubble paths during a pumping cycle by 'seeding' the water flow with air bubbles in the valve seat area near the vena contracta where cavitation is most likely to occur. Consideration of air bubbles is not inappropriate as dissolved air will be liberated in the form of bubbles when the local pressure falls below the saturation pressure. (For water at atmosphere pressure, the dissolved air content is around $2 \%$, the precise level depending on temperature.) If this approach were successful it could be used to assess the effect of changes in valve and cylinder geometries.

Thus the first step is to use the package to predict bubble behaviour in a model of the mock pump and to compare this with results from the flow visualisation experiments. This problem is particularly demanding in terms of CFD modelling as it involves a moving boundary with transient, two-phase, turbulent flow. Predicted behaviour is achieved using a coupled simulation which allows for interaction between phases subject to the following assumptions: (i) buoyancy effects and heat and mass transfer are ignored; and (ii) bubbles rebound ideally.

\section{Mesh structure}

The mock pump constructed for the experimental work was represented by a narrow, wedge-shaped, axisymmetric mesh composed of a single layer of cells. The arrangement is shown in Figure 3. 


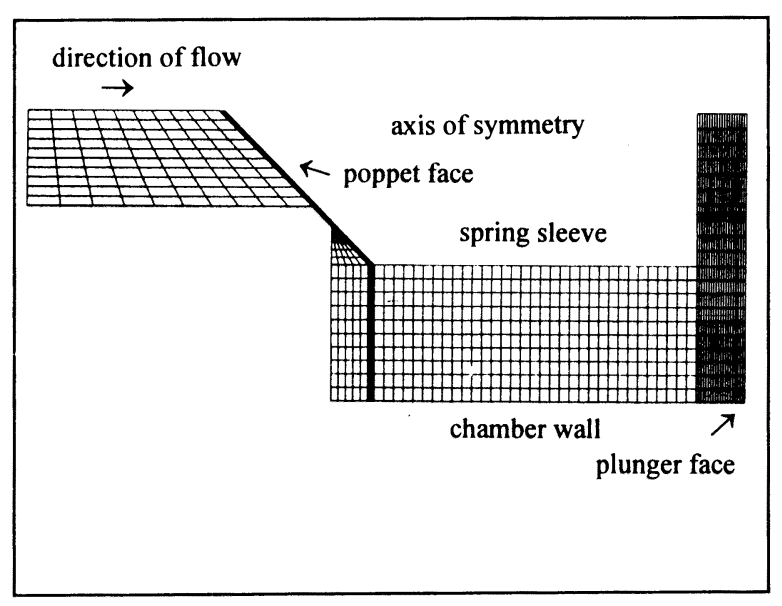

Figure 3. Mesh geometry

The valve spring is covered by a sleeve, corresponding to the experimental arrangement, and the plunger is shown in the top dead centre (TDC) position. In this respect the mesh arrangement deviates from the experimental configuration where the plunger was located some distance downstream of the visualisation chamber. However, the mesh is more representative of a real pumping chamber.

A refined mesh in the region of the poppet valve enabled studies of behaviour with a moving valve, not reported here. The mesh immediately upstream of the plunger face permitted the plunger motion to be simulated through cell expansion and contraction. Plunger motion was assumed to be sinusoidal with the maximum plunger velocity chosen such that the peak flow rate matched that obtained in the experimental tests. The time for the suction stroke was set to $0.12 \mathrm{~s}$, corresponding to the experimental speed of 250 $\mathrm{rev} / \mathrm{min}$. This results in a peak Reynolds number of $5 \times 10^{4}$.

Simulations were run with the plunger moving from TDC to $10^{\circ}$ after bottom dead centre (BDC), with a time step of $0.00926 \mathrm{sec}$ (corresponding to a $2^{\circ}$ change in crank angle). Ideally, the instantaneous valve opening would be established by integrating the pressure distribution over the valve and using the resultant force to solve the equation of motion for the valve mass-spring system. However, this was considered unnecessarily complicated since, in the experiments, the valve opened almost fully in $3 \mathrm{~ms}$. Consequently, predictions were made with a fixed valve opening of $1 \mathrm{~mm}$ (similar to the maximum recorded experimentally).

Early in the suction stroke, a strong jet forms in the vicinity of the valve seat. This is directed out towards the wall of the chamber with a clockwise toroidal vortex forming to the left of the jet due to flow separation at the seat. The vortex remains throughout the suction stroke although its centre moves downstream as the simulation progresses, with the jet tending to move closer to the spring sleeve (although attachment does not occur). An example of predicted velocity vectors at $70^{\circ}$ before BDC is shown in Figure 4. Peak fluid velocity which occurs in the valve seating region is $35 \mathrm{~ms}^{-1}$. The toroidal vortex is clearly identifiable. Around $40^{\circ}$ before BDC a second, but weak vortex begins to develop behind the valve as a result of the velocity field set up by the main vortex.

The flow field remains stable throughout the simulation although the velocity vectors decrease in magnitude until the plunger begins the delivery stroke. Examination of behaviour over the early part of the delivery stroke is not unrealistic since, in practice, there is often a delay after BDC before the inlet valve closes.

Further simulations were performed with a valve with a shorter spring sleeve to provide a more compact arrangement, closer to that in a real pump. The results were very similar in characteristic to those reported above, although the secondary vortex behind the valve was stronger.

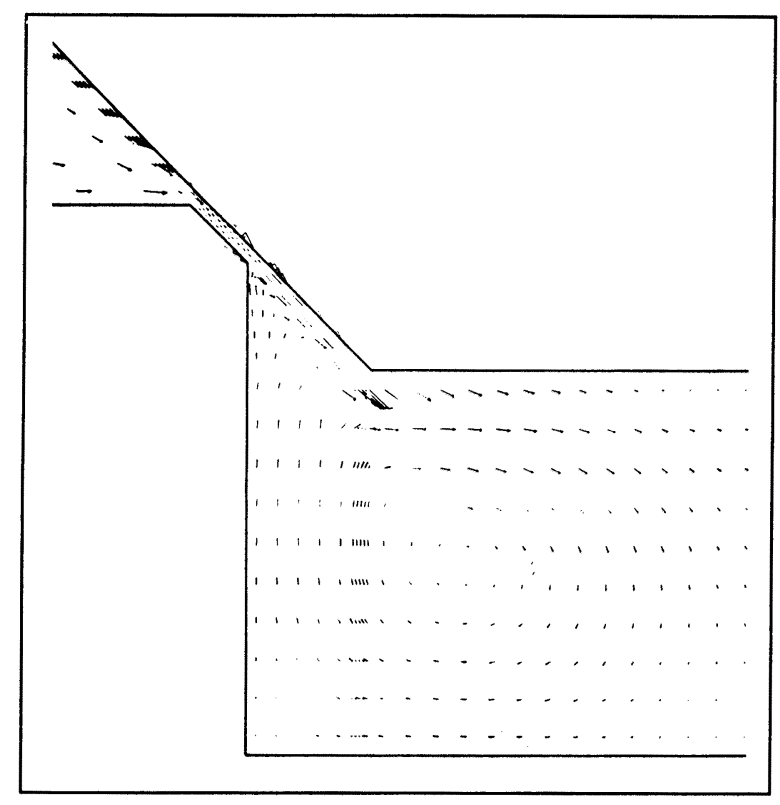

Figure 4. Velocity vectors during suction stroke $-70^{\circ}$ before BDC

\section{INTRODUCTION OF BUBBLES}

Figure 5 indicates the area of bubble 'seeding' that was chosen; these could be introduced at any instant of time during the simulation. 


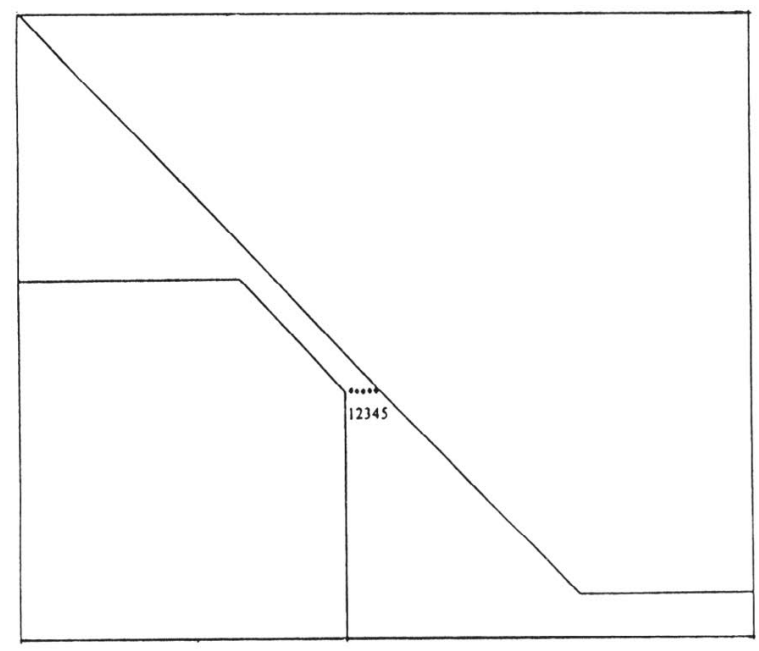

Figure 5. Seeding location for bubbles

Simulations were undertaken to predict the bubble motion during the suction stroke. The initial conditions selected for each bubble were:

- Diameter

$1 \times 10^{-5} \mathrm{~m}$

- Density

$1.205 \mathrm{~kg} / \mathrm{m}^{3}$

- Temperature

$293 \mathrm{~K}$

Bubble density during the simulation is dependent on the local pressure of the water and is calculated assuming that the air has ideal properties. Computational problems arose when the simulations were performed with the supply at atmospheric pressure: these were avoided by pressurising the delivery inlet.

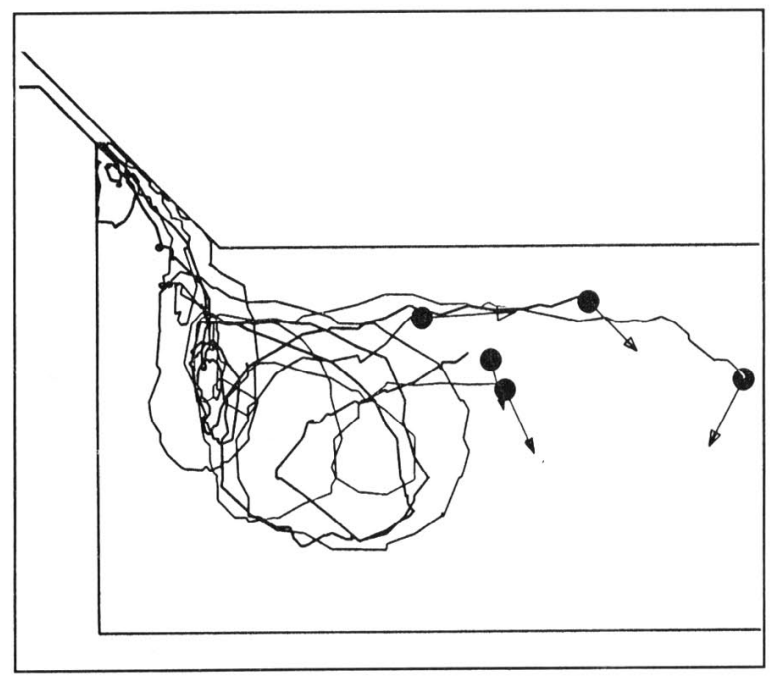

Figure 6. Paths of five bubbles seeded at $5^{\circ}$ after TDC-position shown at $10^{\circ}$ after BDC
Figure 6 shows the predicted paths of five bubbles seeded at $5^{\circ}$ after TDC. From the Figure, which illustrates the paths up to $10^{\circ}$ after BDC, it is apparent that the bubbles followed the flow field, with the movement of the centre of the main vortex being clearly identifiable. Other simulations were conducted with single bubbles [7.] from which it was confirmed that interactions between bubbles had a noticeable effect on the exact paths travelled, although the trends remained unchanged. The location and motion of the bubbles is broadly comparable with that observed experimentally. Figure 7 shows the location of cavities, obtained from flow visualisation, around plunger mid-stroke position. Greatest cavity concentration is in the low-pressure toroidal vortex near the valve, as predicted.



Figure 7. Flow visualisation results

Following these initial simulations, seeding was performed in 'bursts' to represent the effects of oscillations in the valve opening during cavitation, as observed during the flow visualisation studies.

Figure 8 shows the location of 500 bubbles, introduced in bursts at $10^{\circ}$ intervals during the suction stroke, when the plunger has reached a position of $10^{\circ}$ after BDC. The strength of the main vortex is clearly shown, all the bubbles being confined to motion close to the valve and the surrounding walls. The main concentration throughout the cycle is located in the neighbourhood of point A on Figure 8, which corresponds to the location of maximum cavitation in the flow visualisation tests reproduced in Figure 7. This qualitative agreement provided the basis for using the simulation to examine the effect of some changes in geometry. 


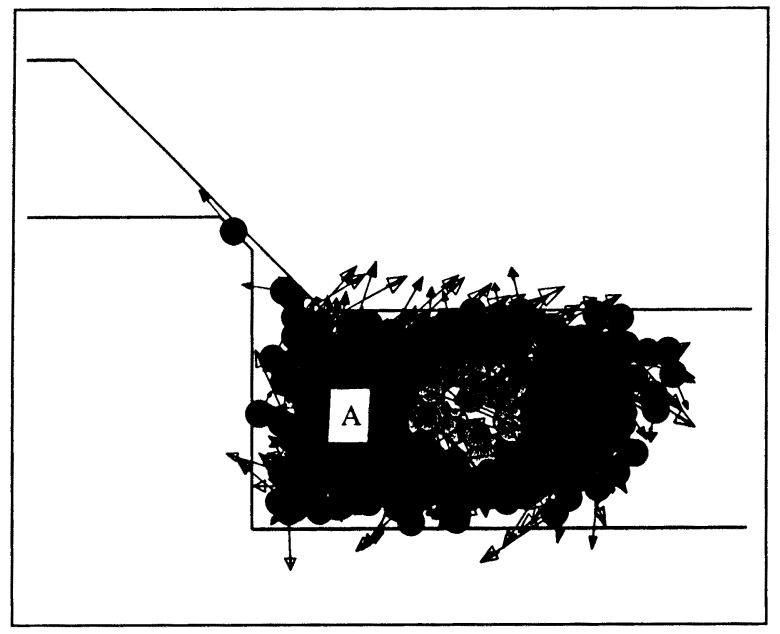

Figure 8. Position of 500 bubbles at $10^{\circ}$ after BDC - original geometry

\section{GEOMETRICAL VARIATIONS}

Figures 9 to 11 show the positions attained by 500 bubbles when the plunger is at $10^{\circ}$ after BDC for three different geometries. Figure 9 shows the results obtained with a change in profile of the plunger head. The face was profiled to assess if this would increase the strength of the secondary vortex behind the valve and thereby draw a proportion of the bubbles away from the valve and the cylinder walls. However, the general pattern of the bubble location is unaffected, principally because the velocity of the fluid at the plunger head was too small to exert any influence on the bubbles.

Figure 10 shows the bubble locations with an extended valve seat. Compared with Figure 8 there is a discernible movement of the bubbles towards the cylinder walls which is undesirable. Although there is a reduction of bubble concentration near the valve which is beneficial.

In Figure 11 the valve face has been modified upstream of the vena contracta combined with an extended valve seat. This has little overall effect compared with Figure 10 apart from removing any bubbles from the vicinity of the valve.



Figure 9. As for Figure 8 with modified plunger face

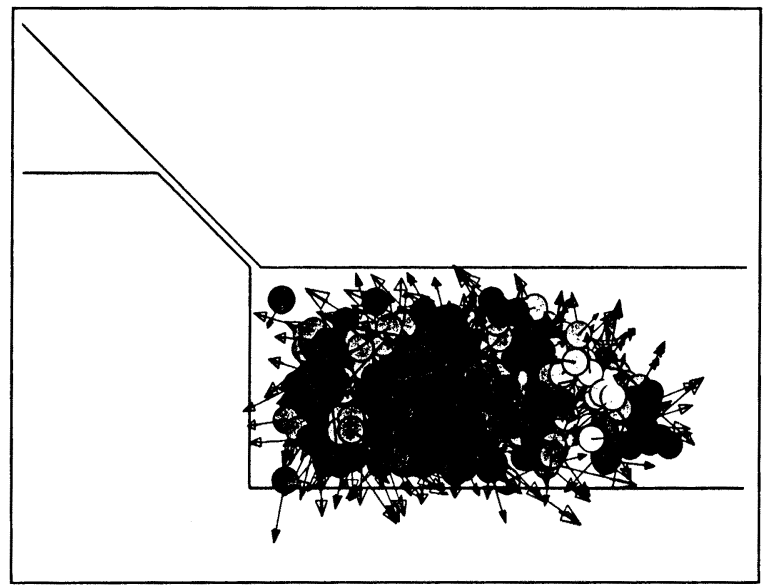

Figure 10. As for Figure 8 with modified valve seat

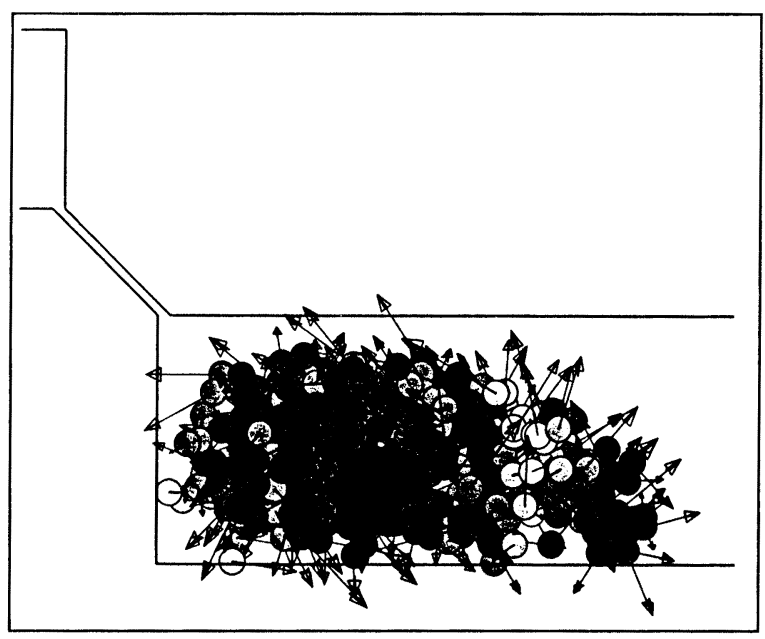

Figure 11. As for Figure 8 with modified valve and valve seat 


\section{CONCLUSIONS}

Computer simulations of a mock plunger pump have shown good correlation with experimental flow visualisation results. The areas of maximum concentration of 'seeded' bubbles, as predicted by the simulation, correspond with the observed area of maximum cavitation. The bubbles are drawn into the strong vortex near the valve.

The computer model showed that changes in the plunger face were not effective in moving the bubbles away from the valve and cylinder walls. An extension of the valve seat is undesirable as it leads to greater bubble concentration near the cylinder wall.

\section{ACKNOWLEDGEMENTS}

The authors wish to acknowledge the support of EPSRC (Grant ref GR/G56423), ICI Engineering, CAT Pumps (UK) Ltd and Dowson Downie Lamont Ltd. Thanks are also expressed to Ford Motor Company Ltd for provision of the STAR-CD program.

\section{REFERENCES}

1. Johnston, D. N., Edge, K. A., Vaughan, N. D. and Raggett, M., Experimental and Computational Study of Pumping Dynamics and Valve Operation in a Reciprocating Pump, ASME Winter Annual Meeting, New Orleans, USA, November 1993, Paper 93-WA/FPST-5.

2. Oshima, S., An Experimental Study on Several Poppet Valves with Difference in Shape, Proceedings of 1st Japan Hydraulics and Pneumatics Society International Symposium on Fluid Power, Editor A. Yamaguchi, Tokyo, 1989.

3. Ishii, Y., Yonezawa, Y., Tsukiji, T., Suzuki, R. and Ishii, S., Flow Visualisation in a Three-Dimensional Poppet Valve, FLUCOME ‘94, Toulouse, France, August/September 1994, pp1095-1100.

4. Ueno, H., Okajima, A. and Muromiya, Y., Visualisation of Cavitating Flow and Numerical Simulation of Flow in a Poppet Valve, In: Fluid Power, Editor T. Maeda, 1993.

5. Edge, K. A., Xiao, S., Burrows, C. R. and Shu, J-J., Flow Visualisation of Cavitation in a Reciprocating Plunger Pump Using High-Speed Cinematography, FLUCOME '94, Toulouse, France, August/September 1994, pp1101-1106.

6. Anon, STAR-CD Manual - Version 2.21, 1995

7. Lecky-Thompson, N., CFD Modelling of Cavitation in a Reciprocating Plunger Pump, Fluid Power Centre Report 039fp/1995, University of Bath, June 1995, 48pp. 